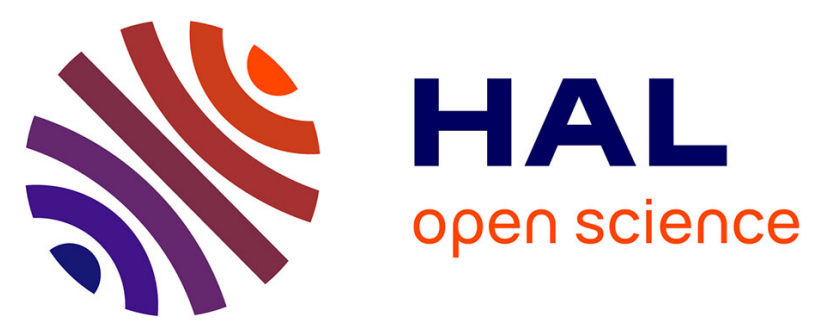

\title{
Backscattering in complex flows: application of the One-Way Euler equations to Poiseuille flow inside lined duct
} Clément Rudel, Sébastien Pernet, Jean-Philippe Brazier

\section{- To cite this version:}

Clément Rudel, Sébastien Pernet, Jean-Philippe Brazier. Backscattering in complex flows: application of the One-Way Euler equations to Poiseuille flow inside lined duct. AIAA AVIATION 2021 FORUM, Aug 2021, VIRTUAL EVENT, United States. 10.2514/6.2021-2138 . hal-03526085

\section{HAL Id: hal-03526085 https://hal.science/hal-03526085}

Submitted on 14 Jan 2022

HAL is a multi-disciplinary open access archive for the deposit and dissemination of scientific research documents, whether they are published or not. The documents may come from teaching and research institutions in France or abroad, or from public or private research centers.
L'archive ouverte pluridisciplinaire HAL, est destinée au dépôt et à la diffusion de documents scientifiques de niveau recherche, publiés ou non, émanant des établissements d'enseignement et de recherche français ou étrangers, des laboratoires publics ou privés. 


\title{
Backscattering in complex flows: application of the One-Way Euler equations to Poiseuille flow inside lined ducts
}

\author{
Clément Rudel*, Sébastien Pernet ${ }^{\dagger}$ and Jean-Philippe Brazier ${ }^{\ddagger}$ \\ ONERA, Université de Toulouse, Toulouse, France, 31400
}

\begin{abstract}
We present an improved formulation of the numerical One-Way approximation that permits to diagonalize in a fully numerical way the propagation operator of a general hyperbolic system. In this paper, it is applied to the linearized Euler equations in the case of a duct with a partially lined section presenting a Poiseuille flow. Domain decomposition is developed in this paper since it is needed for the handling of the transverse boundary conditions discontinuity (hard-wall and impedance condition) which is at the origin of the refraction and reflection of the incident wave inside the computational domain. The results of the One-Way method are compared to experimental and numerical results and show a good accuracy for a low amount of computing resources.
\end{abstract}

\section{Introduction}

The reduction of noise produced by an aircraft is, nowadays, an active field of study since it is a major issue of the development of the commercial aviation worldwide. In particular, we are interested in reducing the sound emitted by aircraft during take off and approach phases since more and more residential area are built close to airports. Acoustic liners are already used inside most of the recent airliners to reduce the sound produced by the engines but their development requires many numerical simulations and experimental setups to be effective. Therefore, computing quickly and easily numerous configurations of liner is an essential issue for the future of the aviation.

The results obtained through experimental setups give invaluable and accurate insights but are undeniably costly, difficult to set up and do not allow to get all the wanted data from a case of study.

On the other hand, numerically, the studied setup is classically modeled with a partially lined duct crossed by (in our case) a subsonic Poiseuille flow. In this configuration, hard wall boundary conditions are imposed at the top all along the duct while the bottom wall is composed of an impedance condition surrounded by hard walls, creating a discontinuity in the computational domain. In this kind of simulations, the main challenges are to model accurately the liner behavior with an impedance condition depending on the frequency and to capture well the reflected and refracted waves coming from the liner leading and trailing edge. Both issues are to be studied carefully in order to obtain accurate results since their effects on the propagation of waves is non negligible and the latter is, in particular, the subject of this paper.

Some methods such as the Direct Numerical Simulation (DNS) or Large Eddy Simulation (LES) [1, 2], are able to give an accurate solution of this wave scattering in all the propagation directions. However, both methods are particularly costly in computational resources and cannot be easily applied for large models or to quickly compute several configurations.

The study of the linear phenomena inside ducts has already shown its potential and already gives good results [3] and takes naturally the reflection and refraction of waves into account but presents some problems. In time domain, some hypothesis are taken such as a uniform flow inside the duct but the impedance condition applied to model the effect of the liner is not straightforward to develop and can lead to numerical instabilities [4]. Therefore, the presence of a shear base flow to linearize the equations can lead to complex developments of impedance and non-reflecting boundary conditions (NRBC). The simulations can also be carried out inside the frequency domain but the problematic remains the same with the need to build boundary conditions that allow the waves propagation outside of the computational domain without reflection. Moreover, inside the frequency domain, this direct resolution method requires to solve a large linear system that can necessitate a large amount of computational resources and CPU time.

On the other hand, we can decide to solve the system locally by performing a spatial resolution along a privileged direction which is imposed by the base flow. The construction of One-Way approximations for hyperbolic systems of equations is precisely based on the uncoupling, in the microlocal sense, between the waves propagating downstream

\footnotetext{
*PhD student, DTIS, clement.rudel@onera.fr

†Dr, DTIS, sebastien.pernet@onera.fr

DDr, DMPE, jean-philippe.brazier@onera.fr
} 
and upstream. This microlocal approach of the One-Way equations has been intensively used in geophysics, acoustics or electromagnetics for almost fifty years, but they have not been applied to more complex systems yet, like Euler or Navier-Stokes, since this method requires approximations of pseudo-differential operators that can be hard to obtain in this context.

Recently, Towne et al. [5] proposed a new formulation which we will call numerical One-Way, in opposition to the analytical one requiring pseudo-differential operators, that showed convincing results when applied to wave propagation in inhomogeneous flows. Based on the concept of non-reflecting boundary condition, this approach makes possible to get rid of the analytical expression of the operators, which permits to apply a One-Way decomposition to more complex systems. However, it still suffers from a limitation: a slow varying hypothesis for the flow along the privileged direction.

We propose to apply the One-Way method (Sec. [II.A) to the case of a lined duct with a sheared flow (Poiseuille). In order to obtain an accurate result on this configuration presenting discontinuities and, therefore, reflection and transmission of the incident wave, a new formulation of the numerical One-Way is developed in Sec. III.B. The computation parameters are presented in Sec. III and the problematic of reflection and transmission of waves is handled in Sec. IV] with the development of a domain decomposition method. Finally, several numerical results are compared to previous computations and experimental data in Sec. $\mathrm{V}$

\section{Method}

\section{A. Linearized Euler equations and numerical One-Way}

The development of the One-way equations will be based on the 2D dimensionless Euler equations. Therefore, we will focus on the formulation of the numerical One-Way as stated by Towne and Colonius [6, 7], but some reviews are available concerning the more standard One-Way formulation [8, 9]. All the variables and coefficients have been made dimensionless by reference values taken from the problem and/or the base flow. The Euler equations used for this method have to be linearized around a base flow and we will use the following decomposition of the variable $q$ :

$$
\mathbf{q}(x, y, t)=\overline{\mathbf{q}}(x, y)+\mathbf{q}^{\prime}(x, y, t),
$$

with $\overline{\mathbf{q}}$ the variable of the steady base flow around which we linearize the equations and $\mathbf{q}^{\prime}$ the fluctuations of this variable that we will compute. In our case, we will assume that $\bar{p}=p_{0}$ constant, $\bar{u}=\bar{u}(x, y)$ and $\bar{v}=0$ and denote the base velocity vector $\overline{\mathbf{U}}=(\bar{u} \bar{v})^{t}$.

Since we are only interested in the stationary behavior of the system, we will consider the Euler equations in a harmonic regime by decomposing the fluctuations into a summation of frequency modes:

$$
\mathbf{q}^{\prime}(x, y, t)=\sum_{N} \widetilde{\mathbf{q}}_{N}(x, y) e^{-i \omega t},
$$

with $\omega$ the wave number.

We will use, for the development of this method, the following 2D isentropic compressible linearized Euler equations:

$$
\left\{\begin{array}{l}
\frac{D \widetilde{\rho}}{D t}+\widetilde{\rho} \nabla \cdot \overline{\mathbf{U}}+\nabla \cdot(\bar{\rho} \widetilde{\mathbf{u}})=0 \\
\bar{\rho} \frac{D \widetilde{\mathbf{u}}}{D t}+\bar{\rho}(\widetilde{\mathbf{u}} \cdot \nabla) \overline{\mathbf{U}}+\widetilde{\rho}(\overline{\mathbf{U}} \cdot \nabla) \overline{\mathbf{U}}+\nabla \widetilde{p}=0 \\
\widetilde{p}=a^{2} \widetilde{\rho}
\end{array},\right.
$$

with $\frac{D}{D t}$ the transport operator which can be written in harmonic regime: $-i \omega+\overline{\mathbf{U}} \cdot \nabla$. We will assume for the rest of this paper that $\bar{\rho}$ and $a$, the speed of sound have a constant value and that we will only work with the primitive variables $\widetilde{p}, \widetilde{u}$ and $\widetilde{v}$. Therefore, the variable vector $\widetilde{q}$ can be written:

$$
\widetilde{\mathbf{q}}=\left(\begin{array}{l}
\widetilde{p} \\
\widetilde{u} \\
\widetilde{v}
\end{array}\right) .
$$

Then, by isolating the $\frac{\partial}{\partial x}$ part, we can put Equation 3 into the following matrix form: 


$$
A(x, y) \frac{\partial \widetilde{\mathbf{q}}}{\partial x}=B(x, y) \frac{\partial \widetilde{\mathbf{q}}}{\partial y}+C(x, y) \widetilde{\mathbf{q}},
$$

with:

$$
A=\left(\begin{array}{ccc}
\bar{u} & a^{2} \bar{\rho} & 0 \\
\frac{1}{\bar{\rho}} & \bar{u} & 0 \\
0 & 0 & \bar{u}
\end{array}\right) ; B=\left(\begin{array}{ccc}
0 & 0 & -a^{2} \bar{\rho} \\
0 & 0 & 0 \\
-\frac{1}{\bar{\rho}} & 0 & 0
\end{array}\right) ; C=\left(\begin{array}{ccc}
i \omega & 0 & 0 \\
0 & i \omega & -\bar{u}_{y} \\
0 & 0 & i \omega
\end{array}\right),
$$

with the subscript $y$ denoting the derivative in $y$ of the base flow variable. This expression of the Linearized Euler equations is simplified due to the fact that we will study in this case a constant Poiseuille flow presenting variations only in the $y$-direction for the axial velocity. From now on, for the sake of readability, the variable $\widetilde{\mathbf{q}}$ will be denoted $\mathbf{q}$.

We proceed to a variable change by diagonalizing the matrix $A$ in order to have access to the characteristic variables $\phi=\left(\begin{array}{lll}\phi^{1} & \phi^{2} & \phi^{3}\end{array}\right)^{t}$. We introduce the transformation matrix $T$ and its inverse $T^{-1}$ to diagonalize the matrix $A$ in the following way:

$$
T^{-1} A T=\widetilde{A}=\left(\begin{array}{cc}
\widetilde{A}_{+} & 0 \\
0 & \widetilde{A}_{-}
\end{array}\right)
$$

with $\widetilde{A}$ be the diagonal matrix containing all the eigenvalues of $A$ which are all real since the system of equation is hyperbolic. The matrices $\widetilde{A}_{+}$and $\widetilde{A}_{-}$contain the positive and negative eigenvalues on their diagonal, respectively. In our particular case, since our base flow velocity is subsonic and positive, we have:

$$
\widetilde{A}_{+}=\left(\begin{array}{cc}
\bar{u}+a & 0 \\
0 & \bar{u}
\end{array}\right) \text { and } \widetilde{A}_{-}=\bar{u}-a .
$$

It can be noticed that, in the case where $\bar{u}=0$, a singularity can appear in the system, making $\widetilde{A}$ non-invertible. This issue is not within the scope of this paper and the method to deal with it can be find in [10]. The eigenvectors matrices of $A$ can also be easily obtained:

$$
T=\left(\begin{array}{ccc}
1 & 0 & 1 \\
a \bar{\rho} & 0 & -a \bar{\rho} \\
0 & 1 & 0
\end{array}\right) \text { and } T^{-1}=\left(\begin{array}{ccc}
\frac{1}{2} & \frac{1}{2 a \bar{\rho}} & 0 \\
0 & 0 & 1 \\
\frac{1}{2} & -\frac{1}{2 a \bar{\rho}} & 0
\end{array}\right) .
$$

Since constant values of $\bar{\rho}$ and $a$ are assumed, the eigenvectors matrix $T$ and its inverse $T^{-1}$ do not depend of $x$ or $y$. These matrices are also used to perform the variable change between $\mathbf{q}$ and $\boldsymbol{\phi}$ following the relation $\boldsymbol{\phi}=\mathbf{T}^{-1} \mathbf{q}$. Then, we can put Equation(5) under the following form:

$$
\frac{\partial \phi}{\partial x}=M(x, y) \phi
$$

with $M(x, y)=\widetilde{A}^{-1} T^{-1}\left(B(x, y) \partial_{y}+C(x, y)\right) T$. The matrix $M$ can be denoted as the propagation operator and its expression is:

$$
M(x, y)=\left(\begin{array}{ccc}
\frac{1}{\bar{u}+a} & 0 & 0 \\
0 & \frac{1}{\bar{u}} & 0 \\
0 & 0 & \frac{1}{\bar{u}-a}
\end{array}\right)\left(\begin{array}{ccc}
i \omega & -\frac{\bar{\rho} a^{2}}{2} \partial_{y}-\frac{\bar{u}_{y}}{2 a \bar{\rho}} & 0 \\
-\frac{\partial_{y}}{\bar{\rho}} & i \omega & -\frac{\partial_{y}}{\bar{\rho}} \\
0 & -\frac{\bar{\rho} a^{2}}{2} \partial_{y}+\frac{\bar{u}_{y}}{2 a \bar{\rho}} & i \omega
\end{array}\right),
$$

with $\partial_{y}$ be the derivative operator $\frac{\partial}{\partial y}$ in $y$.

The particularity of the numerical One-Way approach over the microlocal one [11] is that the transverse discretization of the system appears before the diagonalization of the propagation operator. It will allow us to separate the right-going waves from the left-going ones without analytically building the dispersion relation, which is difficult to do with a system like the Euler equations. It can be noted that the transverse boundary conditions like wall or slip conditions, impedance or non reflective boundary conditions have to be implemented in the system during this step (Sec. [III.B. All 
the transversally discretized vectors and matrices valued operators and variables will be denoted by bold characters. However, even if the discretization of the equation will make us lose the track of the analytical terms during the diagonalization of $M$, the separation of the characteristic variables into two sets depending on the sign of $\widetilde{A}$ is still guaranteed and it will be used for the decomposition of the propagation operator. Therefore, $\phi=\left(\phi_{+} \quad \phi_{-}\right)^{t}$ with the subscript + denotes the downstream modes while - the upstream ones. The number of discretized points of the vectors $\boldsymbol{\phi}_{ \pm}$is also given by the subscripts: + denotes $N_{+}$points while - designates $N_{-}$points. In our case, we can deduce that $N_{+}=2 N_{y}$ and $N_{-}=N_{y}$ with $N_{y}$ the number of discretization points in the transverse direction since $\widetilde{A_{+}}$is a $2 \times 2$ matrix while $\widetilde{A}_{-}$is a scalar function. We can also note that $\phi_{+}=\left(\begin{array}{ll}\phi^{1} & \phi^{2}\end{array}\right)^{t}$ while $\phi_{-}=\phi^{3}$.

The same operation of separation can be applied to the propagation operator $\mathbf{M}$ depending on the wave propagation direction. The equation (10) can be written such as:

$$
\frac{\partial}{\partial x}\left(\begin{array}{l}
\phi_{+} \\
\phi_{-}
\end{array}\right)=\left(\begin{array}{ll}
\mathbf{M}_{++} & \mathbf{M}_{+-} \\
\mathbf{M}_{-+} & \mathbf{M}_{--}
\end{array}\right)\left(\begin{array}{l}
\phi_{+} \\
\phi_{-}
\end{array}\right)
$$

with the first subscript denoting the number of rows $\left(N_{+}\right.$or $\left.N_{-}\right)$and the second, the number of columns of the block matrix.

The discretized operator $\mathbf{M}$ can also be diagonalized following an eigenvalue decomposition with the transformation $\mathbf{V}$ and its inverse $\mathbf{U}$ such as:

$$
\mathbf{M}=\mathbf{V D U},
$$

with $\mathbf{D}$ the diagonal matrix containing all the eigenvalues of $\mathbf{M}$ and $\mathbf{V}$ the corresponding right eigenvectors matrix or $\mathbf{U}$ the corresponding left eigenvectors matrix.

A new variable change can be performed, using this decomposition, defined by $\boldsymbol{\phi}=\mathbf{V} \boldsymbol{\psi}=\boldsymbol{\phi}=\sum_{j=1}^{N_{+}+N_{-}} \psi_{j} \mathbf{v}_{j}$ with $\mathbf{v}_{j}$ the $j$-th eigenvector of $\mathbf{V}$. We keep once again the separation made during the first diagonalization with the + and subscript and equation (12) becomes:

$$
\frac{\partial}{\partial x}\left(\begin{array}{l}
\psi_{+} \\
\psi_{-}
\end{array}\right)=\left(\begin{array}{cc}
\mathbf{D}_{+} & 0 \\
0 & \mathbf{D}_{-}
\end{array}\right)\left(\begin{array}{l}
\psi_{+} \\
\psi_{-}
\end{array}\right)+\mathbf{U} \frac{\partial \mathbf{V}}{\partial x}\left(\begin{array}{l}
\psi_{+} \\
\psi_{-}
\end{array}\right)
$$

Here, the matrix $\mathbf{W}=\mathbf{U} \frac{\partial \mathbf{V}}{\partial x}$, is the refraction/reflection matrix and it couples the functions $\psi_{+}$and $\psi_{-}$together. This coupling would make this system ill-posed for a resolution using a spatial marching algorithm in $x$. This is why this term is neglected in the construction of the numerical One-Way to decouple the left and right going information. Neglecting this term is mandatory to resolve a well-posed system, but it is also at the origin of the slow varying flow hypothesis [7] along the privileged direction ( $x$ in our case). Equation 14 is now reduced to:

$$
\frac{\partial}{\partial x}\left(\begin{array}{l}
\psi_{+} \\
\psi_{-}
\end{array}\right)=\left(\begin{array}{cc}
\mathbf{D}_{+} & 0 \\
0 & \mathbf{D}_{-}
\end{array}\right)\left(\begin{array}{l}
\psi_{+} \\
\psi_{-}
\end{array}\right)
$$

It can be noted that the decoupling procedure does not require a complete diagonalization of the matrix $\mathbf{M}$ and it can be realized by a less fine spectral decomposition where only the eigenspaces are identified. In other words, the construction of the One-Way method can be based on a factorization of $M$ where the matrix $\widetilde{\mathbf{D}}$ can be a block diagonal matrix.

The One-Way method is based on the fact that during the computation, the upstream propagating waves are neglected. We can enforce that by setting the variable $\psi_{-}=\mathbf{0}$. This condition can be reversed if we want to compute the leftgoing modes by applying $\boldsymbol{\psi}_{+}=\mathbf{0}$. Therefore, the One-Way system takes the following form:

$$
\left\{\begin{array}{rl}
\frac{\partial \psi_{+}}{\partial x} & =\mathbf{D}_{+} \psi_{+} \\
\boldsymbol{\psi}_{-} & =0
\end{array},\right.
$$

which can be written in term of characteristic variables: 


$$
\left\{\begin{array}{rl}
\frac{\partial \boldsymbol{\phi}_{+}}{\partial x} & =\mathbf{M}_{++} \boldsymbol{\phi}_{+}+\mathbf{M}_{+-} \boldsymbol{\phi}_{-} \\
\boldsymbol{\phi}_{-} & =\mathbf{U}_{--}^{-1} \mathbf{U}_{-+} \boldsymbol{\phi}_{+}
\end{array} .\right.
$$

The eigenvalues decomposition of the operator $\mathbf{M}$ in $\mathbf{D}, \mathbf{V}$ and $\mathbf{U}$ is really costly to perform and it has to be computed for each station in $x$ if the flow is varying along the $x$-axis. This is why Equations (14) and (15) are only here to show the mechanisms of this method and the origin of its limitation. Therefore, as introduced in [7, 12], a non-reflective boundary condition [13, 14] has been used under the form of a recursive relation in order to approximate the second equation of system (17) which becomes:

$$
\left\{\begin{aligned}
\frac{\partial \boldsymbol{\phi}_{+}}{\partial x} & =\mathbf{M}_{++} \boldsymbol{\phi}_{+}+\mathbf{M}_{+-} \boldsymbol{\phi}_{-} \\
\left(\begin{array}{c}
\boldsymbol{\phi}_{+}^{N_{\beta}} \\
\mathbf{0}
\end{array}\right) & =\underbrace{\prod_{j=0}^{N_{\beta}-1}\left(\mathbf{M}-i \beta_{-}^{j} \mathbf{I}\right)^{-1}\left(\mathbf{M}-i \beta_{+}^{j} \mathbf{I}\right)}_{\mathbf{Z}^{r} \text { : Non-reflection matrix }}\left(\begin{array}{c}
\boldsymbol{\phi}_{+} \\
\boldsymbol{\phi}_{-}
\end{array}\right),
\end{aligned}\right.
$$

with $\boldsymbol{\phi}_{+}^{N_{\beta}}$ an auxiliary variable and $\mathbf{Z}^{r}$ the non-reflection matrix for a right One-Way where the right-going waves are computed while the left-going ones are neglected. Then, the discretized system (18) can be efficiently solved by a spatial marching algorithm in $x$. The coefficient $\beta_{ \pm}$are to be chosen arbitrarily, but with some care. In order to be efficient, the choice of parameters has to put the $\beta_{+}$coefficient near the cluster of rightgoing modes of the operator $\mathbf{M}$ while the $\beta_{-}$ones are to be chosen near the leftgoing modes cluster. Moreover, the more these two sets of coefficients are far from each other, the better the convergence is. Their role is to separate the eigenspace generated by the downstream eigenvectors from the eigenspace generated by the upstream ones. The required insights on the spectrum of $\mathbf{M}$ to chose these sets can be acquired through a simplified local linear stability analysis of the operator.

\section{B. New diagonalization of the operator}

Since our problem presents some variations that are only due to the discontinuity in the transverse boundary condition, the reflected and transmitted waves will not be computed by taking into account the refraction/reflection matrix $\mathbf{W}$. Instead, we will use the eigenvectors matrices $\mathbf{U}$ and $\mathbf{V}$ to decompose the incident wave into its rightgoing and leftgoing components (Sec. IV). Therefore, if we want to take into account these phenomena, we need to express our problem through the $\psi_{ \pm}$variables which are not accessible through the current formulation of the numerical One-Way. In order to do that, the NRBC used for the numerical One-Way will be used again, but this time in the right-going and left-going directions permitting us to well separate the two different eigenspaces generated. Most of the following construction will be based on the right One-Way formalism but it is easy to build the same for a left One-Way by interchanging the + and - parts of the matrices.

The first step is to build our transformation matrices $\mathbf{U}$ and $\mathbf{V}$. In order to do that, we will use the same recursive relation as in the numerical One-Way to form the $\mathbf{Z}^{r}$ matrix. Using the last equation of system (18) to express $\phi_{-}$using $\mathbf{Z}^{r}$ and $\phi_{+}$, we obtain:

$$
\boldsymbol{\phi}_{-}=\left(\mathbf{Z}_{--}^{r}\right)^{-1} \mathbf{Z}_{-+}^{r} \boldsymbol{\phi}_{+} .
$$

This relation can be compared to the last relation of system (17) to obtain the following equality:

$$
\left(\mathbf{Z}_{--}^{r}\right)^{-1} \mathbf{Z}_{-+}^{r} \approx\left(\mathbf{U}_{--}\right)^{-1} \mathbf{U}_{-+} .
$$

For the rest of the construction, we assume that the choice and the number of $\beta_{ \pm}$coefficient is taken to ensure a good enough convergence to replace the symbol $\approx$ by $=$. The same relation can be obtained using $\mathbf{Z}^{l}$ and the -- and -+ parts of $\mathbf{U}$.

Since the matrix $\mathbf{Z}$ built for the right or the left One-Way is composed by the matrix $\mathbf{M}$, it is easy to see that it has the same eigenvectors matrices $\mathbf{V}$ and $\mathbf{U}$ leading to the following relation:

$$
\left(\begin{array}{ll}
\mathbf{U}_{++} & \mathbf{U}_{+-} \\
\mathbf{U}_{-+} & \mathbf{U}_{--}
\end{array}\right)\left(\begin{array}{ll}
\mathbf{Z}_{++} & \mathbf{Z}_{+-} \\
\mathbf{Z}_{-+} & \mathbf{Z}_{--}
\end{array}\right)=\mathbf{F}(\mathbf{D}) \mathbf{U},
$$


with $\mathbf{F}(\mathbf{D})$ the diagonal matrix composed of the reflection coefficient generated by the NRBC applied to the eigenvalues of M. Formally, these reflection coefficients make $\mathbf{F}\left(\mathbf{D}_{+, \mathbf{i}}\right) \rightarrow \infty$ and $\mathbf{F}\left(\mathbf{D}_{-, \mathbf{i}}\right) \rightarrow 0$ when using the matrix $\mathbf{Z}^{r}$ and $\mathbf{F}\left(\mathbf{D}_{+, \mathbf{i}}\right) \rightarrow 0$ and $\mathbf{F}\left(\mathbf{D}_{-, \mathbf{i}}\right) \rightarrow \infty$ when using the matrix $\mathbf{Z}^{l}$. The symbol $\rightarrow$ indicates that $N_{\beta}$ tends to $+\infty$ and that the coefficients are well-placed. Then, we can deduce the following relation:

$$
\operatorname{Span}\left\{\mathbf{U}_{i, *} \in \mathbb{C}^{N_{+}+N_{-}} \mid i=N_{+}+1, \ldots, N_{+}+N_{-}\right\} \in \operatorname{ker} \mathbf{Z}^{r},
$$

meaning that the - eigenvectors of $\mathbf{U}$ are in the kernel of $\mathbf{Z}^{r}$. In the same way, we can easily show that the + eigenvectors of $\mathbf{U}$ are in the kernel of $\mathbf{Z}^{l}$. Using the converged relation 20, into 21, we can deduce, for the right One-Way:

$$
\left\{\begin{array}{l}
\mathbf{Z}_{-+}^{r} \mathbf{Z}_{++}^{r}+\mathbf{Z}_{--}^{r} \mathbf{Z}_{-+}^{r}=\mathbf{0} \\
\mathbf{Z}_{-+}^{r} \mathbf{Z}_{+-}^{r}+\mathbf{Z}_{--}^{r} \mathbf{Z}_{--}^{r}=\mathbf{0}
\end{array} .\right.
$$

Once again, these relations show that the vectors inside $\mathbf{Z}_{-+}^{r}$ and $\mathbf{Z}_{--}^{r}$ are also in the kernel of $\mathbf{Z}^{r}$ in the same way as the - eigenvectors of $\mathbf{U}$ are in this kernel. This leads to:

$$
\operatorname{Span}\left\{\mathbf{Z}_{i, *}^{r} \in \mathbb{C}^{N_{+}+N_{-}} \mid i=N_{+}+1, \ldots, N_{+}+N_{-}\right\} \in \operatorname{ker} \mathbf{Z}^{\mathbf{r}} .
$$

The same conclusion can be made for $\mathbf{Z}^{l}$ whose kernel contains the vectors generated by $\mathbf{Z}_{++}^{l}$ and $\mathbf{Z}_{+-}^{l}$. By using these two results, we can build a new matrix $\widetilde{\mathbf{U}}$ composed of the relevant parts of $\mathbf{Z}^{r}$ and $\mathbf{Z}^{l}$ that generates an approximation of the same eigenspace as $\mathbf{U}$ and equally separated in its rightgoing and leftgoing contributions. This matrix has the form:

$$
\widetilde{\mathbf{U}}=\left(\begin{array}{ll}
\mathbf{Z}_{++}^{\mathbf{l}} & \mathbf{Z}_{+-}^{\mathbf{l}} \\
\mathbf{Z}_{-+}^{\mathbf{r}} & \mathbf{Z}_{--}^{\mathbf{r}}
\end{array}\right) .
$$

This newly formed matrix of "pseudo eigenvectors" permits a new diagonalization of $\mathbf{M}$ following:

$$
\widetilde{\mathbf{U}} \mathbf{M} \widetilde{\mathbf{V}}=\widetilde{\mathbf{D}},
$$

with $\widetilde{\mathbf{D}}$ a diagonal block matrix. From that, we can build an approximation of the One-Way system 16 by replacing $\mathbf{D}$ with its approximate $\widetilde{\mathbf{D}}$.

The additional computation time induced by the construction of the "pseudo eigenvectors" matrix and the refraction/reflection term, compared to the standard numerical One-Way, is of around 50 to $60 \%$ on this part of the code. Indeed, since both formulations share the same spatial marching algorithm and the construction of the discretized system, no extra computational time is induced by the rest of the method.

\section{Application to lined ducts}

The case of a lined duct presenting a sheared flow has been chosen for this application of the One-Way since we want to compute a case that is well-documented and has experimental comparison data. Moreover, it is a case where the variation along $x$ does not come from the flow but from the boundary conditions and more particularly, this variation is a discontinuity inside the discretized propagation operator $\mathbf{M}$. Therefore, it is a case where the information brought by the One-Way diagonalization is of vital interest since it will be used to decompose the incident wave propagating through such discontinuity into a reflected and transmitted wave.

\section{A. Configuration of the lined duct}

Two different configurations are studied here and they have been chosen in order to apply the One-Way method to a certain number of different cases. The first one is developed in [15] for the experimental results and in [16] for the selected numerical ones and it will be referred to as the Configuration A from now on. The second configuration can be found in [17] for the experimental as well as the numerical results and it will be denoted Configuration B. Both configurations are very similar and can be described by the Fig. 1. However, some differences can be found in term of base flow velocity, dimensions and liner characteristics.

Concerning the base flow, the same laminar fully developed Poiseuille flow will be used in both configurations but with different values of $M_{\max }$. This flow is modeled by the following equation [17]:

$$
\bar{u}(y)=4 M_{\max } a \frac{y}{h}\left(1-\frac{y}{h}\right),
$$




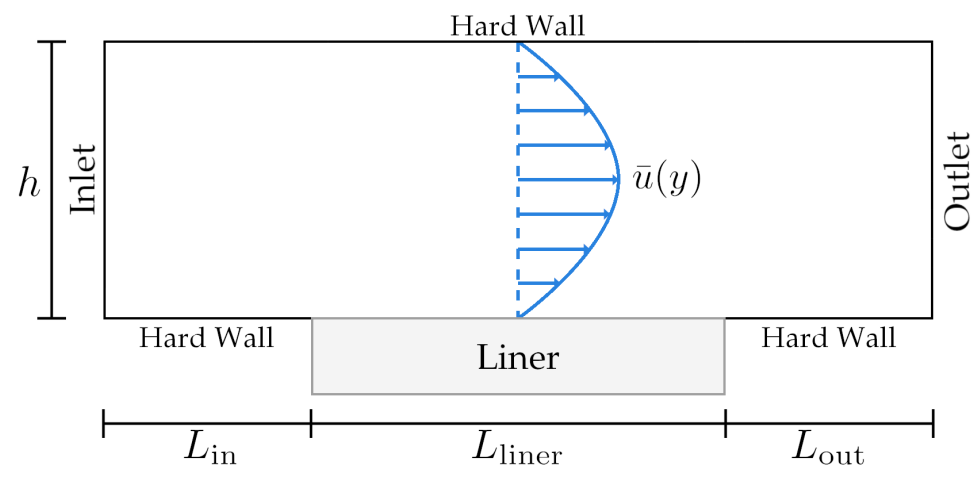

Fig. 1 Configuration of the lined duct

with $M_{\max }$ the maximum value of the Mach number inside the duct, equal to 0.508 for the Configuration A while it is set to 0.3 in Configuration B.

The physical dimensions of the computational domain are also different due to the fact that we base our computations on existing experimental installations. Table 1 shows the particular dimensions of each configuration.

\begin{tabular}{lcc}
\hline & Configuration A & Configuration B \\
\hline$h$ & $0.051 \mathrm{~m}$ & $0.0508 \mathrm{~m}$ \\
$L_{\text {in }}$ & $0.203 \mathrm{~m}$ & $0.210 \mathrm{~m}$ \\
$L_{\text {liner }}$ & $0.203 \mathrm{~m}$ & $0.387 \mathrm{~m}$ \\
$L_{\text {out }}$ & $0.203 \mathrm{~m}$ & $0.243 \mathrm{~m}$ \\
Liner & CT57 & CT73 \\
\hline
\end{tabular}

Table 1 Configuration dimensions

For both configurations, the simulations have been made dimensionless by choosing $L_{\text {ref }}=h, V_{\text {ref }}=340 \mathrm{~m} / \mathrm{s}$ and $\rho_{\text {ref }}=1.23 \mathrm{~kg} / \mathrm{m}^{3}$ as reference values.

Finally, the numerical parameters used for all the simulations are more or less the same. In $y$, the mesh is composed of 50 points evenly spaced (unless mentioned otherwise) and 500 points in $x$ with an exponential distribution to have a refined mesh around the discontinuities. This mesh has been chosen to have a well discretized mesh of 60 points per wavelength on average, in $x$, for the case presenting the highest frequency. The transverse discretization is done by a 6-th order compact finite differences scheme [18] while the space marching algorithm is performed using 1st order Discontinuous Galerkin method, which has the advantage to be unconditionally stable to solve this transport equation [19] while remaining affordable in term of computational cost.

\section{B. Transverse boundary conditions}

The duct has hard walls at the top and the bottom, but a discontinuity of material appears at the bottom with an acoustic liner that presents an impedance condition. This discontinuity is the origin of the reflection and refraction phenomena appearing in these simulations.

\section{Hard wall}

Hard wall boundary conditions are applied at the top and the bottom of the duct and allow the particles to slip tangentially to the wall while imposing the normal velocity $(v)$ to 0 . Moreover, this slip boundary condition models a infinitely thin acoustic boundary layer [20] and satisfies the following set of equations: 


$$
\left\{\begin{array}{l}
\frac{\partial p}{\partial t}+\bar{u} \frac{\partial p}{\partial x}+\bar{\rho} a^{2}\left(\frac{\partial u}{\partial x}+\frac{\partial v}{\partial y}\right)+S a \frac{\partial p}{\partial y}=0 \\
\frac{\partial u}{\partial t}+\bar{u} \frac{\partial u}{\partial x}+\frac{1}{\bar{\rho}} \frac{\partial p}{\partial x}=0 \\
\frac{\partial v}{\partial t}+\bar{u} \frac{\partial v}{\partial x}=0
\end{array},\right.
$$

with $S=1$ or $S=-1$ for the upper or lower wall, respectively. Because we discretize our propagation operator in term of characteristic variables, we have to express this relation in these variables as well. Since this set of equations does not change anything to the diagonalization of the matrix $A$, the transformation matrices $T$ and $T^{-1}$ are also the same as previously. The same can be said about $\widetilde{A}$ and the eigenvalues of $A$. Therefore, by applying the same variable change performed for the Euler equations in this case, we obtain a modified propagation operator $\widetilde{M}$ :

$$
\widetilde{M}(x, y)=\left(\begin{array}{ccc}
\frac{1}{\bar{u}+a} & 0 & 0 \\
0 & \frac{1}{\bar{u}} & 0 \\
0 & 0 & \frac{1}{\bar{u}-a}
\end{array}\right)\left(\begin{array}{ccc}
i \omega+\frac{S a}{2} \partial_{y} & -\frac{\bar{\rho} a^{2}}{2} \partial_{y} & \frac{S a}{2} \partial_{y} \\
0 & i \omega & 0 \\
\frac{S a}{2} \partial_{y} & -\frac{\bar{\rho} a^{2}}{2} \partial_{y} & i \omega+\frac{S a}{2} \partial_{y}
\end{array}\right) .
$$

This new propagation operator is valid and applied only for the $\phi$ variables that are modeling the behavior of the hard wall boundary condition.

\section{Liner}

The presence of the acoustic liner is modeled by an impedance condition that couples the pressure fluctuation with the normal velocity fluctuation since there exists some flow through the liner, contrary to the hard wall boundary condition. This impedance condition is applied following the classical relation:

$$
\widetilde{p}=Z(\omega) \widetilde{\mathbf{u}} \cdot \mathbf{n},
$$

with $\mathbf{n}$ the unitary outward pointing normal at the wall and $Z(\omega)$ the frequency dependent complex impedance which means, in our case, that its value has to be modified according to the value of $\omega$ set. For Configuration A, the value of $Z$ respects the extended Helmholtz resonator relation:

$$
Z(\omega)=R-i m \omega+i \Phi \cot \left(\omega h+i \frac{\epsilon}{2}\right),
$$

with $R$ the face-sheet resistance, $m \omega$ the face-sheet reactance, $\Phi$ a porosity parameter, $h$ the cavity depth and $\epsilon$ the damping in the cavity's fluid. Each of these parameters is imposed by the type of liner used [21] which is here a CT57. Therefore, for our particular computation, a value of $Z=5.21-1.07 i$ is chosen at a frequency of $f=2000 \mathrm{~Hz}$.

For Configuration B, the impedance values are obtained through a nonlinear least-square fit of the experimental data and the different values used between 500 and $3000 \mathrm{~Hz}$ are available in [22].

Since the discretization of our system is made in term of characteristic variables, we have to express Equation (30) in this variables, which gives us:

$$
\phi^{1}+\phi^{3}=Z(\omega) \phi^{2} .
$$

This equation is imposed on the second equation which transports $\phi^{2}$ while the other two equations remain unchanged.

\section{Domain decomposition and transmission of waves}

The presence of the liner at the bottom of the duct creates a discontinuity for the One-Way method since it will use a spatial marching algorithm to solve the equations along $x$. Therefore, an incident wave propagating through such a discrepancy will produce a transmitted wave, propagating in the same direction as before with modified wavelength and amplitude, and a reflected one, propagating in the opposite direction. Splitting the computational domain into 3 different subdomains (in our case) that are completely homogeneous in $x$ independently leads us to compute the reflected and transmitted part of an incident wave only at the interface of these subdomains (Sec. IV.B. 
However, to take into account these phenomena, solving the One-Way equations in only one direction is not sufficient to obtain an accurate result since a non negligible part of the waves can propagate in the opposite direction. Hence it is required to perform a rightgoing One-Way (right OW) to capture the incident wave and its transmission but also a leftgoing one (left OW) to take into account the reflected waves (Sec. IV.A.)

\section{A. Domain decomposition and convergence}

In our particular case, the computational domain $\Omega$ will be split in three different subdomains $\Omega=\left\{\Omega_{1}, \Omega_{2}, \Omega_{3}\right\}$. Each of these subdomains is non-overlapping with another but has one or two interfaces positioned exactly at the liner leading and/or trailing edge. The reflected and transmitted waves will be communicated from a subdomain to another through these interfaces and their computation will be developed in the next section (Sec. IV.B).

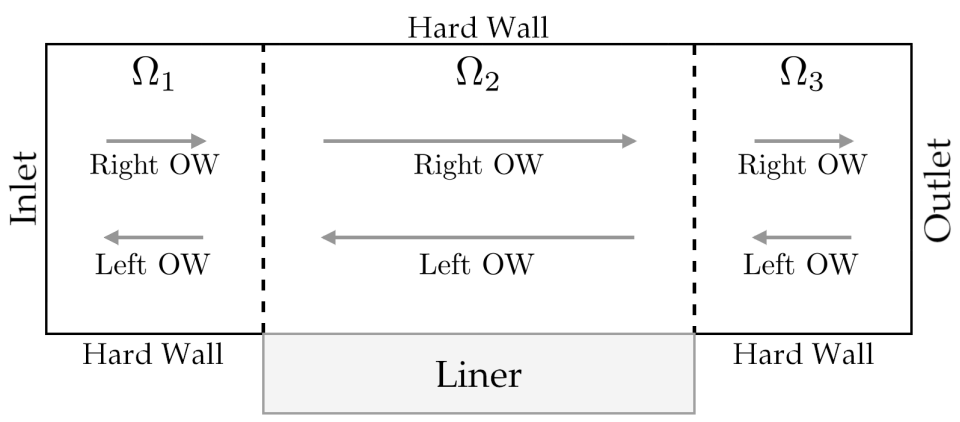

Fig. 2 Decomposition of the computational domain

The layout of Fig 2 is identical for all the simulations performed in this paper, since their configurations are nearly the same. In each subdomain, a right and left One-Way are computed to capture the rightgoing and leftgoing waves, respectively. Once this first approximation of the results is obtained, the reflection and refraction of waves is computed at each interface before updating the initial condition of each One-Way (left and right). A new iteration is then computed, giving a new result that is closer to the final result since it takes into account the reflected and transmitted waves of the previous approximation. These iterations can be carried on until a satisfying convergence is reached.

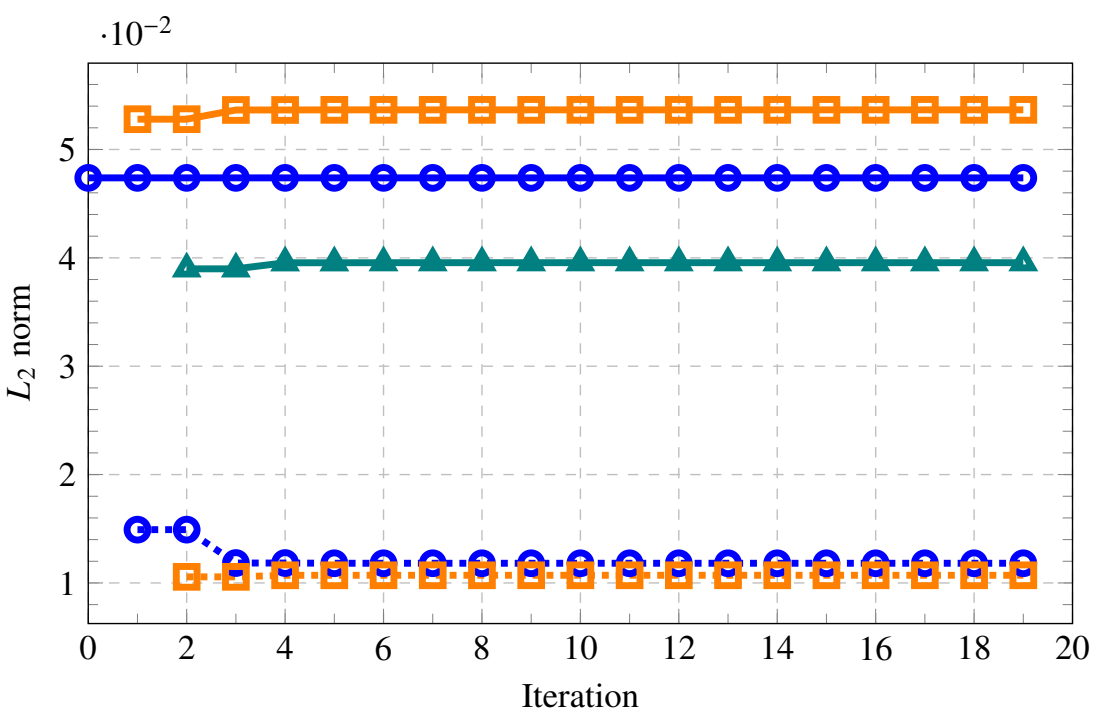

Fig. $3 L_{2}$ norm of the left and right One-Way in each domain depending on the iteration number. - is for the right One-Way, ... is for left One-Way. $\mathcal{O}$ are for $\Omega_{1}, \square$ are for $\Omega_{2}$ and $\Delta$ are for $\Omega_{3}$

We can see in Fig. 3 the $L_{2}$ norm of each iteration of the left and right One-Way in each subdomain for the case at $f=500 \mathrm{~Hz}$ in Configuration B (Sect. III.A. This case has been initialized with a local mode of a hard-walled duct 
(plane wave) to obtain a pure rightgoing wave at the inlet. At iteration 0 , only the right $\mathrm{OW}$ in $\Omega_{1}$ is visible since it is the only one that has a non-null initialization vector (incident rightgoing wave). In the same way, it is expected for its norm to stay the same throughout the iterations since this initialization is not modified. The same analysis can be made about the left OW inside $\Omega_{3}$ because of its initialization with a null vector meaning that no leftgoing wave goes inside our computational domain from the outlet. The norm of the rightgoing OW in $\Omega_{2}$ and leftgoing OW in $\Omega_{1}$ first appears at iteration 1 due to the fact that the incident wave at the inlet has to travel through $\Omega_{1}$ at iteration 0 before being transmitted and reflected. Similarly, the norm of the rightgoing OW in $\Omega_{3}$ and the leftgoing OW in $\Omega_{2}$ take a different value from zero at iteration 2 for the exact same reason. Finally, it can be noticed that the norm of each One-Way in each domain is stabilized to its final value after 4 or 5 iterations, which are necessary for the reflected waves to travel in all subdomains, meaning that our transmission condition is a good approximation of the exact operator.

\section{B. Transmission of waves}

Before communicating the reflected and transmitted waves to other subdomains, we first need to compute which part of the incident wave leaving the current subdomain is reflected back in it and which part actually leaves it before being transmitted to the next domain. To ensure the continuity of our final result even through a discontinuity, we want to impose the following relation at the interface:

$$
\mathbf{q}_{1}=\mathbf{q}_{2},
$$

with $\mathbf{q}_{1}=\left(\begin{array}{lll}p_{1} & u_{1} & v_{1}\end{array}\right)^{t}$ the discretized variables in $\Omega_{1}$ at the interface and $\mathbf{q}_{2}=\left(\begin{array}{lll}p_{2} & u_{2} & v_{2}\end{array}\right)^{t}$ the same variables at the interface but in $\Omega_{2}$. For the rest of this development, the under-script $i$ means that the variable belongs to $\Omega_{i}$ at the interface with $\Omega_{i+1}$ or $\Omega_{i-1}$. The example of the transmission is taken between $\Omega_{1}$ and $\Omega_{2}$ here but it is also relevant for any other domain having a transmission interface. This condition can present some convergence issues for wave transmission problem and more complex conditions are usually employed for this kind of concern [23]. However, the fact that, thanks to the numerical diagonalization of the propagation operator, we are able to decompose a wave into its rightgoing and leftgoing components ensure us a well-posed condition. To decompose these waves and make our transmission problem accurate, we need to express Equation (33) in term of the One-Way variables $\psi_{1}$ and $\psi_{2}$. To do that, we use first the characteristic variables:

$$
\mathbf{T}_{1} \phi_{1}=\mathbf{T}_{2} \phi_{2} .
$$

Since, in our case, the switch in boundary conditions does not lead to a discontinuity in $\mathbf{T}_{i}\left(\right.$ i.e. $\left.\mathbf{T}_{1}=\mathbf{T}_{2}\right)$, we can simplify Equation 34 into:

$$
\phi_{1}=\phi_{2} .
$$

This leaves us, in term of One-Way variables:

$$
\mathbf{V}_{1} \psi_{1}=\mathbf{V}_{2} \psi_{2} .
$$

By decomposing $\mathbf{V}_{i}$ in its + and - components, we obtain:

$$
\left\{\begin{array}{c}
\mathbf{V}_{1}^{++} \psi_{1}^{+}+\mathbf{V}_{1}^{+-} \boldsymbol{\psi}_{1}^{-}=\mathbf{V}_{2}^{++} \boldsymbol{\psi}_{2}^{+}+\mathbf{V}_{2}^{+-} \boldsymbol{\psi}_{2}^{-} \\
\mathbf{V}_{1}^{-+} \boldsymbol{\psi}_{1}^{+}+\mathbf{V}_{1}^{--} \boldsymbol{\psi}_{1}^{-}=\mathbf{V}_{2}^{-+} \boldsymbol{\psi}_{2}^{+}+\mathbf{V}_{2}^{--} \boldsymbol{\psi}_{2}^{-}
\end{array} .\right.
$$

We now have an expression of our original relation in Equation $\sqrt{33}$ in term of leftgoing and rightgoing components. This decomposition allows us to impose only rightgoing information as the transmitted wave and leftgoing information as the reflected wave. The last step is to express directly the wanted terms. In Fig. 4, we can see a diagram of the situation we are in for this refraction/reflection between $\Omega_{1}$ and $\Omega_{2}$. After performing a right One-Way in $\Omega_{1}$, we get an incident rightgoing wave $\psi_{1}^{+}$while the left One-Way in domain $\Omega_{2}$ gives us $\psi_{2}^{-}$. These variables are approximations of the final incident waves but are enough to make $\psi_{1}^{+}$and $\psi_{2}^{-}$known values leaving us with two equations for two unknowns.

We can easily express the missing values $\psi_{2}^{+}$and $\psi_{1}^{-}$from $\psi_{1}^{+}$and $\boldsymbol{\psi}_{2}^{-}$, which gives: 


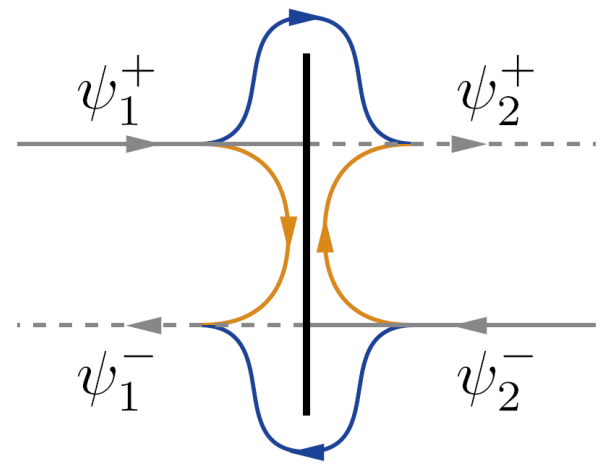

Fig. 4 Transmission of wave through a discontinuity

$$
\left\{\begin{array}{l}
\boldsymbol{\psi}_{2}^{+}=\left(\mathbf{V}_{2}^{++}-\mathbf{V}_{1}^{+-}\left(\mathbf{V}_{1}^{--}\right)^{-1} \mathbf{V}_{2}^{-+}\right)^{-1}\left(\left(\mathbf{V}_{1}^{++}-\mathbf{V}_{1}^{+-}\left(\mathbf{V}_{1}^{--}\right)^{-1} \mathbf{V}_{1}^{-+}\right) \psi_{1}^{+}-\left(\mathbf{V}_{2}^{+-}-\mathbf{V}_{1}^{+-}\left(\mathbf{V}_{1}^{--}\right)^{-1} \mathbf{V}_{2}^{--}\right) \psi_{2}^{-}\right) \\
\boldsymbol{\psi}_{1}^{-}=\left(\mathbf{V}_{1}^{--}\right)^{-1}\left(\mathbf{V}_{2}^{-+} \boldsymbol{\psi}_{2}^{+}+\mathbf{V}_{2}^{--} \psi_{2}^{-}-\mathbf{V}_{1}^{-+} \boldsymbol{\psi}_{1}^{+}\right)
\end{array}\right.
$$

Then, we can set the newly obtained values as initialization for the left One-Way in $\Omega_{1}$ and for the right One-Way in $\Omega_{2}$. By applying this relation at each iteration and at each interface, we are able to compute more and more accurate approximations of the reflected and transmitted waves, converging towards the exact result.

\section{Numerical results}

The numerical results presented here have been computed on the two different configurations presented above (Sec. III.A. To compare the obtained results with the One-Way with experimental data and other numerical results, we have to introduce the sound pressure level (SPL):

$$
S P L=20 \log \left(\frac{|p|}{p_{\text {ref }}}\right),
$$

with $p_{\text {ref }}=2 \times 10^{-5} \mathrm{~Pa}$ the reference sound pressure. The SPL is expressed in decibels $(\mathrm{dB})$.

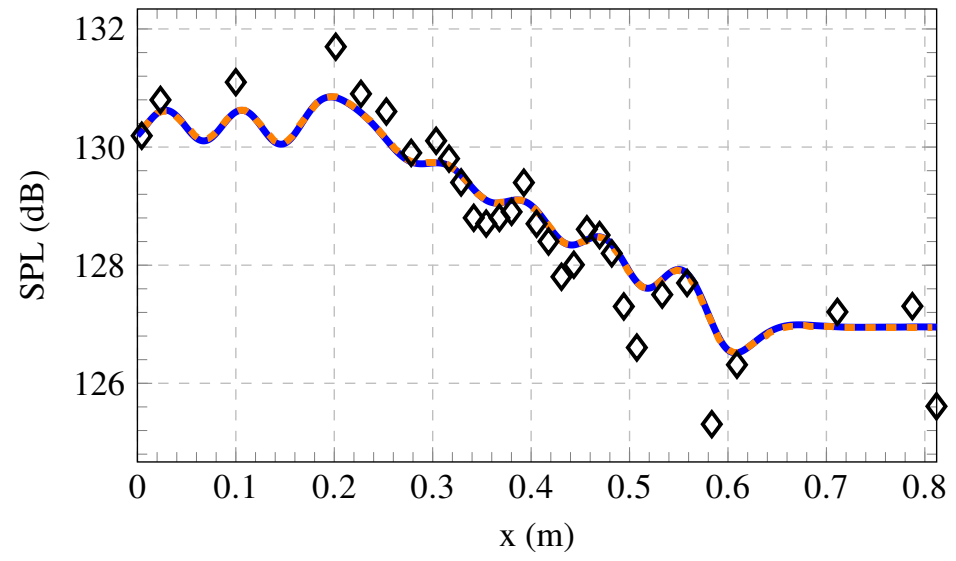

Fig. 5 Sound Pressure Level along the upper wall for Configuration A. Comparison between experimental data (冈) from [15], time domain simulation $[-\infty$ from [16] and the One-Way simulations with domain decomposition

In both cases, the One-Way simulations have been initialized by a plane wave mode that has been scaled to correspond to the same SPL as the numerical data. These plane waves have been obtained through a Local Stability Analysis 
performed at the inlet of the computational domain and by selecting the eigenvectors corresponding to the plane wave mode of the operator spectrum.

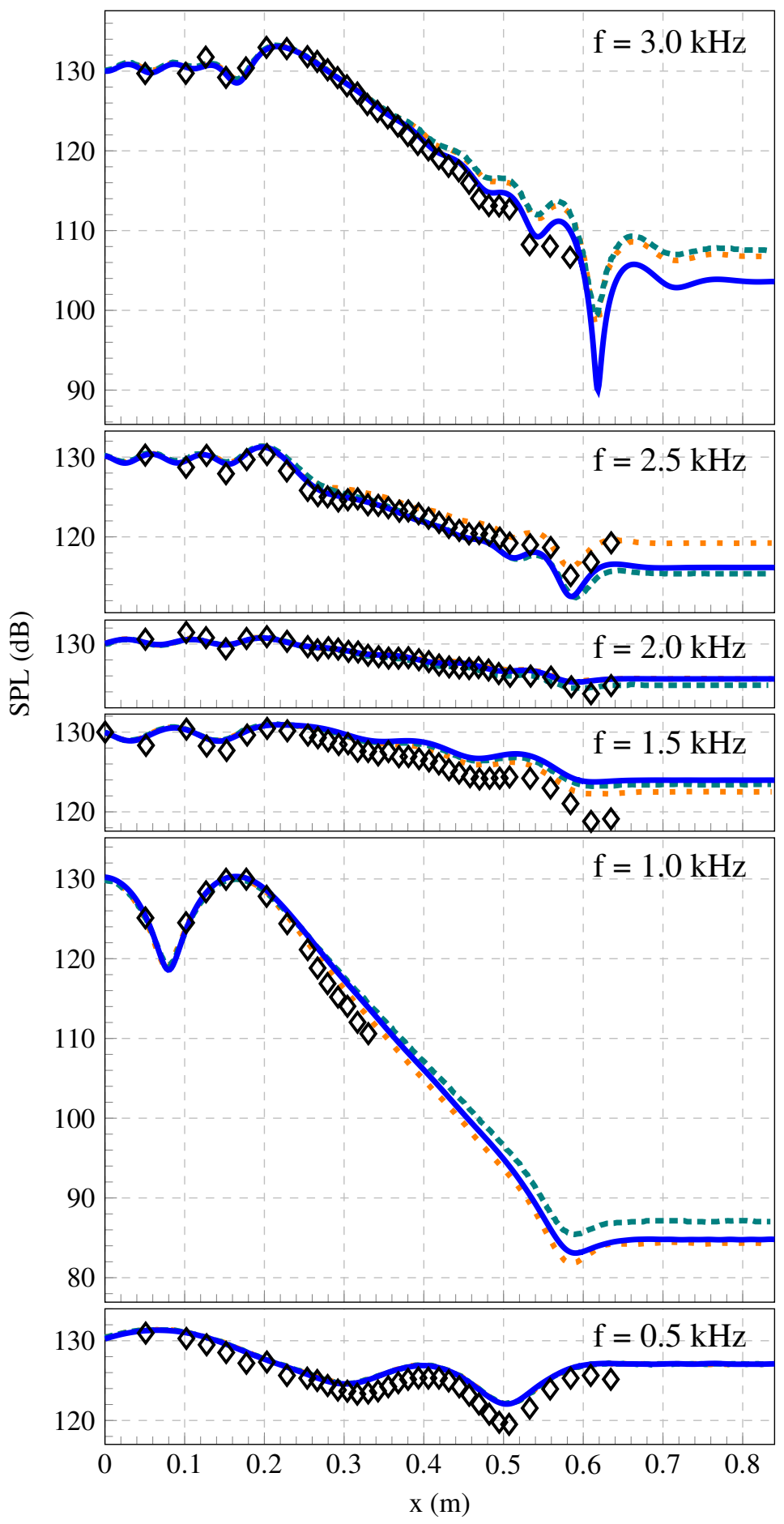

Fig. 6 Sound Pressure Level along the upper wall for Configuration B. Comparison between experimental data $(\nabla)$, multifrequency $(-\cdots)$ and single frequency $\because m$ from $[17]$ and the One-Way simulations with domain decomposition $\longrightarrow$ 
Configuration A For the first configuration, we will refer to numerical results that can be found in [16] and experimental data obtained in [15]. The numerical simulation has been carried out globally and in the time domain, contrary to our local solution computed in the frequency domain.

We can see in Fig. 5 the SPL obtained at the upper wall all along the computational domain. It is clear that the One-Way results are quite close to the other numerical results. The fact that these SPL are really close to each other means that the reflection and refraction of wave at the discontinuity (i.e. at the leading and trailing edge of the liner) are accurately captured and propagated since the global computation takes naturally into account these phenomena. In term of computational cost, this One-Way simulation was performed on only two $2.60 \mathrm{GHz}$ Xeon processors with a total CPU time of 90 seconds while only 4 iterations of One-Way were needed for the domain decomposition to converge.

Configuration B The second configuration has for particularity to be set at $M_{\max }=0.3$, which is slower than in the previous case where $M_{\max }=0.5$. Moreover, the One-Way simulations have been carried out at different frequencies, from $f=500 \mathrm{~Hz}$ to $f=3000 \mathrm{~Hz}$, every $500 \mathrm{~Hz}$ to explore the validity domain of such method in this type of application. All the numerical and experimental comparison data are taken from [17]. Once again, these results have been obtained through a global time domain computation with a Fast Fourier Transform (FFT) applied to the solution in order to get the SPLs at the upper wall.

In Fig. 6, the SPL along the upper wall is shown for every frequency studied here. It is directly visible that the One-Way results are quite close to either the multifrequency source simulation or the single frequency source one in most of the cases. In particular, at $f=1500 \mathrm{~Hz}$, where a deviation between the numerical result and the experimental data was noticed [17] for an unknown reason, the same deviation with the One-Way seems to appear. On the contrary, this very same difference at $f=3000 \mathrm{~Hz}$ is less important in the case of our OW simulation. The same analysis can be made for Configuration A and B about the reflected and refracted waves that are well captured since both the rightgoing waves at the outlet and the leftgoing waves at the inlet gives an accurate result that is in accordance with the global simulation. The CPU time required for these simulations is the same as for the previous configuration with 90 seconds of total CPU time. However, the case at $f=1000 \mathrm{~Hz}$ required the implementation of a compact 4th order finite differences scheme in the transverse direction for a non uniform mesh [18], since oscillations in the SPL appeared after the liner with a constant step grid. Therefore, this discretization scheme allows a more refined mesh at the hard wall and liner boundary conditions, making these oscillations disappear with only 100 points in $y$ and 600 seconds of total CPU time. The reason behind the difference of results for the multi and single frequency source simulations might be the fact that with an incident wave presenting a SPL of around $130 \mathrm{~dB}$, some areas inside the simulations might have a higher SPL that could lead to a nonlinear behavior of the computed waves.

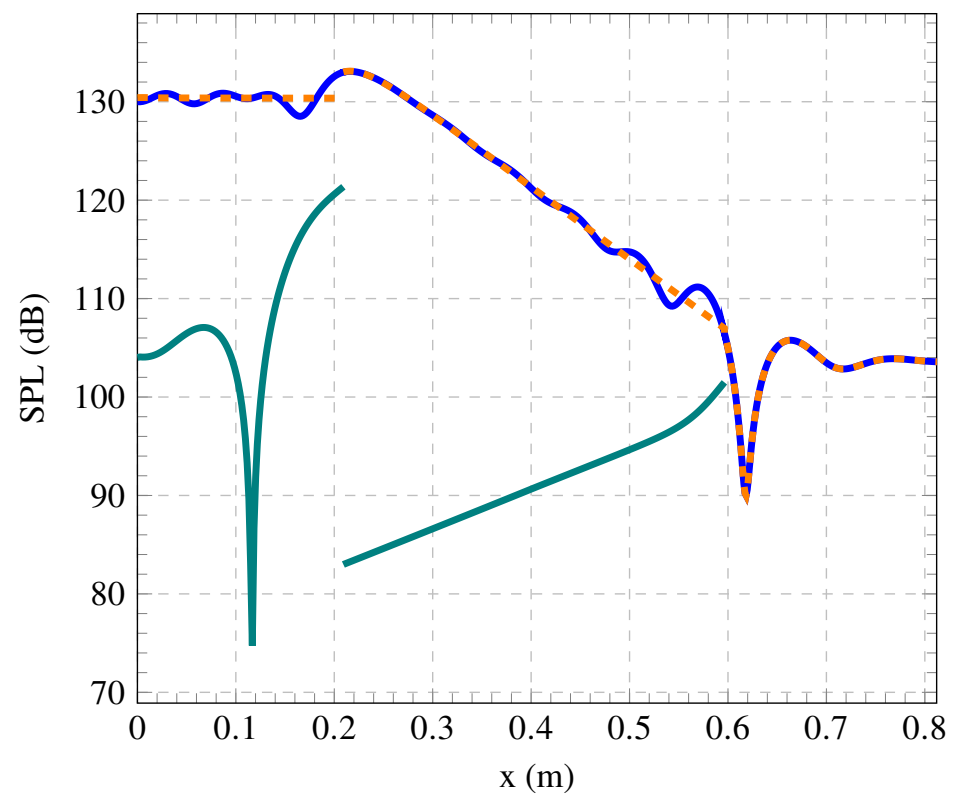

Fig. 7 Sound Pressure Level along the upper wall for Configuration B at $f=3000 \mathrm{~Hz}$. Decomposition of the final result $\square$ into its rightgoing $\square=$ and its leftgoing $\square$ components 
One of the particular feature of the One-Way method is that it is possible to decompose our result into its rightgoing and leftgoing contributions. In the same way as previously, Fig. 7 shows the SPL at the upper wall for the case $f=3000 \mathrm{~Hz}$ along with the SPL of the leftgoing and rightgoing components. We can see that the reflected waves, with a maximum SPL of $120 \mathrm{~dB}$ are not negligible compared to the maximum SPL of the rightgoing waves that is of $133 \mathrm{~dB}$, even if we speak here of a logarithmic scale. Moreover, the oscillations present on the liner and before it are entirely the results of these reflected waves since they can not be observed on the contribution propagating to the right. In the end, the SPL of the wave propagating downstream is of $103 \mathrm{~dB}$ which is lower than the SPL of the outgoing wave propagating upstream with a value of $104 \mathrm{~dB}$. It can be noticed that the SPL after the liner is null for the leftgoing waves, as expected, since nothing is imposed at the outlet and no reflection can occur after the second discontinuity.

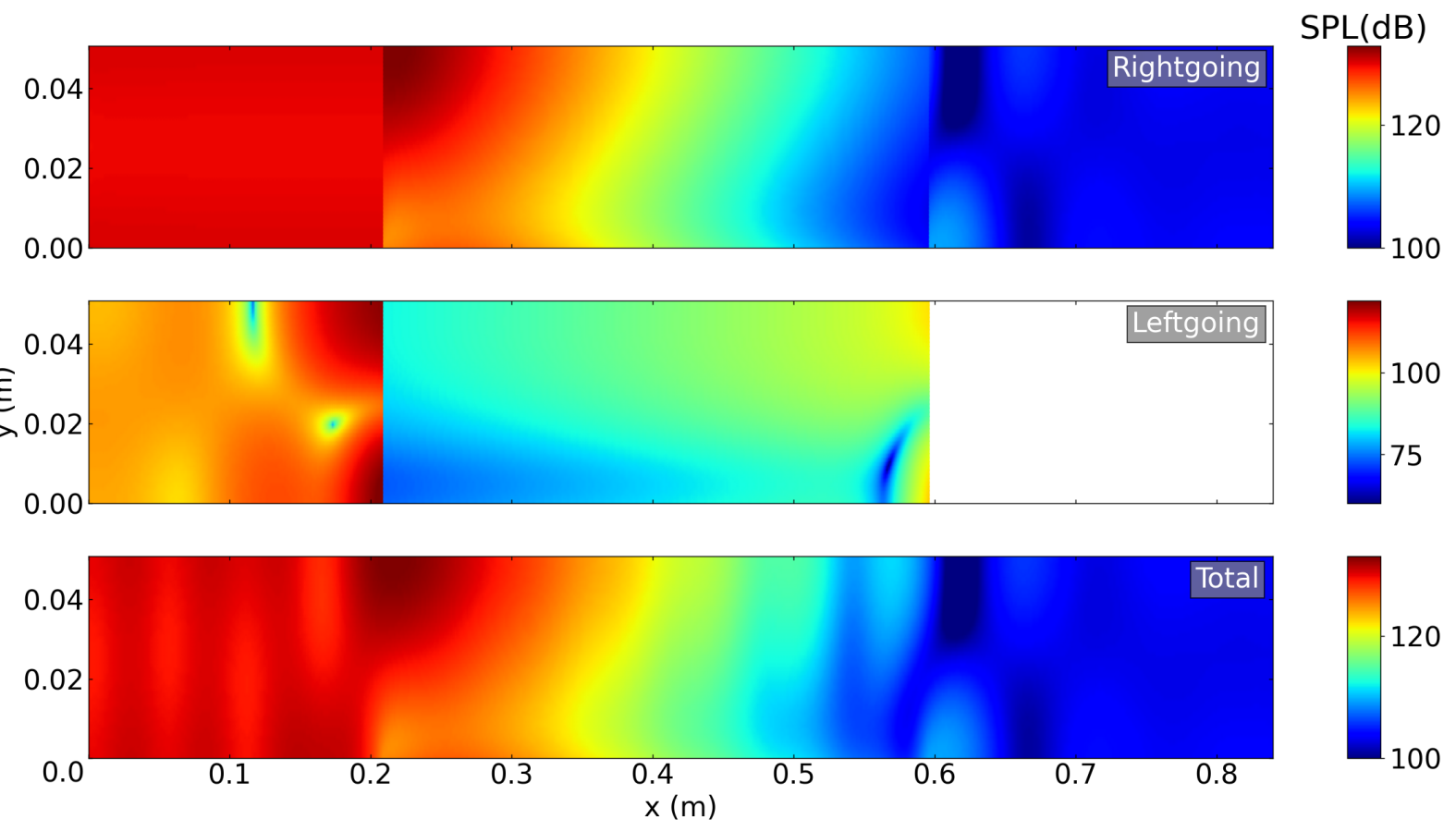

Fig. 8 Decomposition of the Sound Pressure Level into the rightoing and leftgoing components and total results for Configuration B at $f=3000 \mathbf{H z}$

The SPL on the whole computational domain is presented in Fig. 8. As expected, the SPL of the rightgoing part is homogeneous at a fixed value before the liner before decreasing, with the lowest values at the bottom (where the liner is) before stabilizing after the liner. For the leftgoing part, the SPL is null on the right side of the liner before presenting some strong variations at the bottom of the duct and decreasing in value due to the effect of the acoustic liner. These waves come only from the reflection of the rightgoing part at the liner trailing edge. Between the inlet and the beginning of the liner, the SPL values are bigger due to the fact that the rightgoing part is at around $130 \mathrm{~dB}$ which is more than the $104 \mathrm{~dB}$, on average, present for the second discontinuity. As in Fig. 7) we can see the drop in SPL on the second plot on the upper wall, along with some other in the middle and the bottom of the duct. Discontinuities can be seen in both the downstream and upstream propagating components since only the summation of them guarantees a smooth transition.

\section{Conclusion}

We presented a diagonalization method based on a non-reflective boundary condition that can be applied to a generic hyperbolic system since this decomposition of the propagation operator is fully numerical and does not require heavy analytical developments. In particular, we applied this method to the linearized Euler equations to compute a case of partially lined duct presenting a shear flow. One of the particularities of this kind of configuration is the presence of discontinuities in the boundary conditions along the propagation axis and each of them has been processed to avoid any ill-posedness.

This discontinuity issue has been handled by the projection of waves, leading to their decomposition in rightgoing 
and leftgoing components, which is now possible thanks to the numerical diagonalization of the operator. This feature has been used here to compute the reflected and refracted part of a wave across a discontinuity but it can be used in a more wider range of application, such as domain decomposition in heterogeneous media or preconditioning.

Finally, some numerical results were presented with comparison to existing numerical and experimental data. It seems to give good results, in accordance to the previous existing numerical data for a wide range of velocity and frequency and for a low computational cost. This type of method also allows a decomposition of the final results into rightgoing and leftgoing components, giving more insights on the phenomena appearing in this kind of configurations.

\section{Acknowledgments}

This work was partly supported by the french "Programme d'Investissements d'avenir" ANR-17-EURE-0005 conducted by ANR

\section{References}

[1] Colonius, T., and Lele, S., "Computational Aeroacoustics: Progress on Nonlinear Problems of Sound Generation," Progress in Aerospace Sciences, Vol. 40, 2004, pp. 345-416.

[2] Tam, C., Computational Aeroacoustics: A Wave Number Approach, Cambridge University Press, 2012.

[3] Richter, C., Hay, J. A., Panek, ł., Schönwald, N., Busse, S., and Thiele, F., “A Review of Time-Domain Impedance Modelling and Applications," Journal of Sound and Vibration, Vol. 330, No. 16, 2011, pp. 3859-3873. https://doi.org/10.1016/j.jsv.2011.04.013

[4] Brambley, E. J., "Fundamental problems with the model of uniform flow over acoustic linings," Journal of Sound and Vibration, Vol. 322, No. 4, 2009, pp. 1026-1037. https://doi.org/10.1016/j.jsv.2008.11.021.

[5] Towne, A., and Colonius, T., “One-Way Spatial Integration of Hyperbolic Equations,” Journal of Computational Physics, Vol. 300, 2015, pp. 844-861. https://doi.org/10.1016/j.jcp.2015.08.015

[6] Towne, A., and Colonius, T., "Improved Parabolization of the Euler Equations," 19th AIAA/CEAS Aeroacoustics Conference, AIAA Paper 2013-2171, American Institute of Aeronautics and Astronautics, 05/27-29/2013. https://doi.org/10.2514/6.20132171

[7] Towne, A. S., "Advancements in Jet Turbulence and Noise Modeling: Accurate One-Way Solutions and Empirical Evaluation of the Nonlinear Forcing of Wavepackets," Ph.D. thesis, California Institute of Technology, 2016.

[8] Halpern, L., and Trefethen, L. N., "Wide-angle One-way Wave Equations," The Journal of the Acoustical Society of America, Vol. 84, No. 4, 1988, pp. 1397-1404. https://doi.org/10.1121/1.396586.

[9] Angus, D. A., “The One-Way Wave Equation: A Full-Waveform Tool for Modeling Seismic Body Wave Phenomena," Surveys in Geophysics, Vol. 35, No. 2, 2014, pp. 359-393. https://doi.org/10.1007/s10712-013-9250-2

[10] Majda, A., and Osher, S., "Initial-boundary value problems for hyperbolic equations with uniformly characteristic boundary," Communications on Pure and Applied Mathematics, Vol. 28, No. 5, 1975, pp. 607-675. https://doi.org/10.1002/cpa.3160280504

[11] Halpern, L., and Trefethen, L. N., "Wide-angle one-way wave equations," The Journal of the Acoustical Society of America, Vol. 84, No. 4, 1988, pp. 1397-1404. https://doi.org/10.1121/1.396586 URL https://asa.scitation.org/doi/10.1121/1.396586. publisher: Acoustical Society of America.

[12] Towne, A., and Colonius, T., "Continued Development of the One-Way Euler Equations: Application to Jets," 20th AIAA/CEAS Aeroacoustics Conference, AIAA Paper 2014-2903, American Institute of Aeronautics and Astronautics, 06/16-20/2014. https://doi.org/10.2514/6.2014-2903

[13] Higdon, R. L., "Numerical Absorbing Boundary Conditions for the Wave Equation," Mathematics of Computation, Vol. 49, No. 179, 1987, pp. 65-90. https://doi.org/10.2307/2008250.

[14] Hagstrom, T., and Warburton, T., "A New Auxiliary Variable Formulation of High-Order Local Radiation Boundary Conditions: Corner Compatibility Conditions and Extensions to First-Order Systems," Wave Motion, Vol. 39, No. 4, 2004 , pp. 327-338. https://doi.org/10.1016/j.wavemoti.2003.12.007 
[15] Jones, M., Watson, W., and Parrott, T., "Benchmark Data for Evaluation of Aeroacoustic Propagation Codes with Grazing Flow," 11th AIAA/CEAS Aeroacoustics Conference, Aeroacoustics Conferences, American Institute of Aeronautics and Astronautics, 2005. https://doi.org/10.2514/6.2005-2853.

[16] Pascal, L., Piot, E., and Casalis, G., "A New Implementation of the Extended Helmholtz Resonator Acoustic Liner Impedance Model in Time Domain CAA," Journal of Computational Acoustics, Vol. 24, No. 01, 2015 , p. 1550015. https://doi.org/10.1142/S0218396X15500150

[17] Özyörük, Y., and Long, L. N., “Time-Domain Calculation of Sound Propagation in Lined Ducts with Sheared Flows," AIAA Journal, Vol. 38, No. 5, 2000, pp. 768-773. https://doi.org/10.2514/2.1056

[18] Gamet, L., Ducros, F., Nicoud, F., and Poinsot, T., "Compact Finite Difference Schemes on Non-Uniform Meshes. Application to Direct Numerical Simulations of Compressible Flows," International Journal for Numerical Methods in Fluids, Vol. 29, No. 2, 1999, pp. 159-191. https://doi.org/10.1002/(SICI)1097-0363(19990130)29:2<159::AID-FLD781>3.0.CO;2-9.

[19] Pietro, D. A. D., and Ern, A., Mathematical Aspects of Discontinuous Galerkin Methods, Mathématiques et Applications, Springer-Verlag, Berlin Heidelberg, 2012. https://doi.org/10.1007/978-3-642-22980-0

[20] Nayfeh, A. H., Kaiser, J. E., and Telionis, D. P., "Acoustics of Aircraft Engine-Duct Systems," AIAA Journal, Vol. 13, No. 2, 1975, pp. 130-153. https://doi.org/10.2514/3.49654

[21] Richter, C., Thiele, F. H., Li, X. D., and Zhuang, M., "Comparison of Time-Domain Impedance Boundary Conditions for Lined Duct Flows,” AIAA Journal, Vol. 45, No. 6, 2007, pp. 1333-1345. https://doi.org/10.2514/1.24945.

[22] Özyörük, Y., Long, L. N., and Jones, M. G., “Time-Domain Numerical Simulation of a Flow-Impedance Tube,” Journal of Computational Physics, Vol. 146, No. 1, 1998, pp. 29-57. https://doi.org/10.1006/jcph.1998.5919

[23] Collino, F., Ghanemi, S., and Joly, P., "Domain Decomposition Method for Harmonic Wave Propagation : A General Presentation,” Research Report RR-3473, INRIA, 1998. 